\title{
On Speeding up Frequent Approximate Subgraph Mining
}

\author{
Niusvel Acosta-Mendoza, Andrés Gago-Alonso, and José E. Medina-Pagola \\ Advanced Technologies Application Center (CENATAV), Havana, Cuba. \\ \{nacosta, agago, jmedina\}@cenatav.co.cu
}

\begin{abstract}
Frequent approximate subgraph (FAS) mining has become an interesting task with wide applications in several domains of science. Most of the previous studies have been focused on reducing the search space or the number of canonical form (CF) tests. CF-tests are commonly used for duplicate detection; however, these tests affect the efficiency of mining process because they have high computational complexity. In this paper, two prunes are proposed, which allow decreasing the label space, the number of candidates and the number of CF-tests. The proposed prunes are already used and validated in two reported FAS miners by speeding up their mining processes in artificial graph collections.
\end{abstract}

Keywords: Approximate graph mining, approximate graph matching, frequent approximate subgraphs, labeled graphs.

\section{Introduction}

In recent years, the necessity to use approximate matching in graph mining tasks has increased 7]. In fact, there are concrete problems where exact matching could not be applicable with positive outcome [5]. Sometimes, interesting subgraphs show slight differences throughout datasets. This means that we should tolerate certain level of geometric distortion, slight semantic variations, vertices or edges mismatch in frequent pattern search. For this reason, it is required to evaluate the similarity between graphs allowing some structural differences, i.e. considering approximate matching.

Taking into account these facts, several algorithms have been developed for FAS mining which use different approximate graph matching techniques in different domains of science [1, 4 $6,6,[11]$. However, only APGM [6] and VEAM [1] detect the FASs in a graph collection, where the approximation consists in considering some variations of the data through the substitution probability, keeping the topology of the graphs. These algorithms specify which vertices, edges or labels can replace others. Thus, the idea that not always a vertex label or an edge label can be replaced by any other is defended. APGM only deals with the variations between the vertex labels while VEAM perform the mining process using the vertex and edge label sets.

On the other hand, candidates are represented by a unique code called CF for frequency counting. In order to obtain the occurrences of the candidates are 
performed CF-tests to each of them. However, the computational complexity of a CF-test is very high [3]. In this paper, we introduce several prunes for improving FAS mining in an approximate framework related to APGM and VEAM approaches. Using these prunes it is possible to reduce the label space and the search space, as well as number of candidates. This fact also allows us to reduce the number of CF-tests.

The basic outline of this paper is as follows. Section 2 provides some basic concepts. The prunes for speeding up approximate methods are provided in section 3. The experimental results are discussed in section 4 . Finally, conclusions of the research and some ideas about future directions are exposed in Section 5.

\section{Basic Concepts}

This work is focused on simple undirected labeled graphs. Henceforth, when we refer to graph we assume this type of graph. Before presenting their formal definition, we define the domain of labels.

Let $L_{V}$ and $L_{E}$ be label sets, where $L_{V}$ is a set of vertex labels and $L_{E}$ is a set of edge labels. The domain of all possible labels is denoted by $L=L_{V} \cup L_{E}$.

A labeled graph in $L$ is a 4-tuple, $G=(V, E, I, J)$, where $V$ is a set whose elements are called vertices, $E \subseteq\{\{u, v\} \mid u, v \in V, u \neq v\}$ is a set whose elements are called edges (the edge $\{u, v\}$ connecting the vertex $u$ with the vertex $v), I: V \rightarrow L_{V}$ is a labeling function for assigning labels to vertices and $J: E \rightarrow L_{E}$ is a labeling function for assigning labels to edges.

Let $G_{1}=\left(V_{1}, E_{1}, I_{1}, J_{1}\right)$ and $G_{2}=\left(V_{2}, E_{2}, I_{2}, J_{2}\right)$ be two graphs, we say that $G_{1}$ is a subgraph of $G_{2}$ if $V_{1} \subseteq V_{2}, E_{1} \subseteq E_{2}, \forall u \in V_{1}, I_{1}(u)=I_{2}(u)$, and $\forall e \in E_{1}, J_{1}(e)=J_{2}(e)$. In this case, we use the notation $G_{1} \subseteq G_{2}$ and we say that $G_{2}$ is a supergraph of $G_{1}$.

Given two graphs $G_{1}=\left(V_{1}, E_{1}, I_{1}, J_{1}\right)$ and $G_{2}=\left(V_{2}, E_{2}, I_{2}, J_{2}\right)$, where $G_{1} \subseteq$ $G_{2}$, we say that $e=\{u, v\} \in E_{2}$ is an extension of $G_{1}$ if: $V_{2}=V_{1} \cup\{v\}$ and $E_{1}=E_{2} \backslash\{e\}$. This fact can be denoted by $G_{2}=G_{1} \diamond e$. We say that $e$ is a backward extension if $v \in V_{1}$, otherwise we say that it is a forward extension (it extends the vertex set of $G_{1}$ ).

Given $G_{1}$ and $G_{2}$, we say that $f$ is an isomorphism between these graphs if $f: V_{1} \rightarrow V_{2}$ is a bijective function, where $\forall u \in V_{1}, f(u) \in V_{2} \wedge I_{1}(u)=I_{2}(f(u))$ and $\forall\{u, v\} \in E_{1},\{f(u), f(v)\} \in E_{2} \wedge J_{1}(\{u, v\})=J_{2}(\{f(u), f(v)\})$. When there is an isomorphism between $G_{1}$ and $G_{2}$, we say that $G_{1}$ and $G_{2}$ are isomorphic.

Let $\Omega$ be the set of all possible labeled graphs in $L$, the similarity between two elements $G_{1}, G_{2} \in \Omega$ is defined as a function sim : $\Omega \times \Omega \rightarrow[0,1]$. We say that the elements are very different if $\operatorname{sim}\left(G_{1}, G_{2}\right)=0$, the higher the value of $\operatorname{sim}\left(G_{1}, G_{2}\right)$ the more similar the elements are and if $\operatorname{sim}\left(G_{1}, G_{2}\right)=1$ then there is an isomorphism between these elements.

Let $G_{1}=\left(V_{1}, E_{1}, I_{1}, J_{1}\right), G_{2}=\left(V_{2}, E_{2}, I_{2}, J_{2}\right)$ and $T=\left(V_{T}, E_{T}, I_{T}, J_{T}\right)$ be three labeled graphs in $L$, where $T \subseteq G_{2}$. Using an isomorphism threshold $\tau$, we say that $T$ is an embedding of $G_{1}$ in $G_{2}$ if $\operatorname{sim}\left(G_{1}, T\right) \geq \tau$. The embedding set of $G_{1}$ in $G_{2}$ is denoted by $O\left(G_{1}, G_{2}\right)$. 
Let $T$ be an embedding of $G_{1}$ in $G_{2}=\left(V_{2}, E_{2}, I_{2}, J_{2}\right)$, using an isomorphism threshold $\tau$. The extension set of $T$ is denoted by $\operatorname{ExtSet}(T)=\left\{e \in E_{2} \mid e\right.$ is an extension of $T\}$.

Let $D=\left\{G_{1}, \ldots, G_{|D|}\right\}$ be a graph collection and let $G$ be a labeled graph in $L$, the support value of $G$ in $D$ is obtained through the following equation:

$$
\operatorname{supp}(G, D)=\sum_{G_{i} \in D} \operatorname{sim}\left(G, G_{i}\right) /|D|
$$

If $\operatorname{supp}(G, D) \geq \delta$, then the graph $G$ is approximately frequent in the collection $D$, saying that $G$ is a $F A S$ in $D$. Notice that when we refer to a graph collection we assume that it is the representation built from a real graph collection. The value of the support threshold $\delta$ is in $[0,1]$ assuming that the similarity is normalized to 1. FAS mining consists in finding all the FASs in a collection of graphs $D$, using a similarity function sim and a support threshold $\delta$.

\section{Prunes for Mining}

In this section, we introduce two prunes for FAS mining. These prunes can be used in any FAS miner which uses substitution matrices and they are based on the downward closure property [2]. VEAM and APGM are used as basis for showing the improves.

The candidate generation process consists of extending a subgraph pattern by an edge. This is done searching the possible approximate label set of the new edge $e$ and for every possible label of $e$ seeking the possible label set of the new vertex if $e$ is a forward extension. Finally, candidates are generated using those labels that satisfy definitions "Vertex Approximate Sub-isomorphism 11 " and "Approximate Sub-isomorphism 2]" presented by Acosta-Mendoza et al. [1]. In the above mentioned label sets, there are labels that could replace another ones with a similarity less than the threshold $\tau$. Using these labels it is not possible to obtain candidates; however, the algorithms lose time checking these extensions. To formalize the previous assertions the following definition is presented.

Definition 1 (Useful label set). Let $l_{v} \in L_{V}, l_{e} \in L_{E}$ be a vertex label and an edge label respectively, we say that the label sets, for a vertex label $l_{v}$ and an edge label $l_{e}$, are useful label sets if they are obtained by the functions $U_{V}^{\tau}: L_{V} \rightarrow P_{L_{V}}$ and $U_{E}^{\tau}: L_{E} \rightarrow P_{L_{E}}$ such that:

$$
\begin{aligned}
& -U_{V}^{\tau}\left(l_{v}\right)=\left\{l \in L_{V} \mid \frac{M V_{l, l_{v}}}{M V_{l, l}} \geq \tau\right\}, \\
& -U_{E}^{\tau}\left(l_{e}\right)=\left\{l \in L_{E} \mid \frac{M E_{l, l_{e}}}{M E_{l, l}} \geq \tau\right\} ;
\end{aligned}
$$

where, $P_{L_{V}}$ and $P_{L_{E}}$ are the power sets of $L_{V}$ and $L_{E}$ respectively $3^{3}$.

\footnotetext{
${ }^{1}$ Definition used as similarity function in APGM

${ }^{2}$ Definition used as similarity function in VEAM

${ }^{3}$ For a set $X$, the power set of $X$ is $P_{X}=\{Y \mid Y \subseteq X\}$.
} 
Notice that, for each $l_{v} \in L_{V}, U_{V}^{\tau}\left(l_{v}\right) \neq \emptyset$, since at least $l_{v} \in U_{V}^{\tau}\left(l_{v}\right)$. In the same way, for each $l_{e} \in L_{E}, U_{E}^{\tau}\left(l_{e}\right) \neq \emptyset$, since at least $l_{e} \in U_{E}^{\tau}\left(l_{e}\right)$.

Theorem 2. Let $G$ be a labeled graph, let $M V$ and $M E$ be a substitution matrix indexed by vertex labels and a substitution matrix indexed by edge labels respectively; let $v, v^{\prime}$ be two vertices with labels $l_{v}$ and $l_{v^{\prime}}$ respectively, and let $e=\{u, v\}, e^{\prime}=\left\{u, v^{\prime}\right\}$ be two edges with labels $l_{e}$ and $l_{e^{\prime}}$ respectively. Then the following statements are true:

1. If $l_{v^{\prime}} \notin U_{V}^{\tau}\left(l_{v}\right)$, then $G \diamond e^{\prime} \neq_{A} G \diamond e$ (in the same way $\left.G \diamond e^{\prime} \neq_{a} G \diamond e\right)$.

2. If $l_{e^{\prime}} \notin U_{E}^{\tau}\left(l_{e}\right)$, then $G \diamond e^{\prime} \neq_{A} G \diamond e$.

Proof. In these statements, the definitions of approximate sub-isomorphism of APGM and VEAM are used. These definitions use a product of substitution probabilities with the requirement that its result should be greater than or equal to $\tau$. These substitution probability values are in the interval $[0,1]$. Therefore, if a factor of this product is lesser than $\tau$ then its value is less than $\tau$ too and does not generate an approximate subgraph candidate.

On the other hand, in APGM and VEAM, the approximation is based on the semantic of labels. For this reason, we proposed a heuristic to reduce the search space, according to some rules that can be applied by these FAS miners. These rules are: (1) The vertices (and its corresponding edges), which are not used as an embedding of a single-vertex FAS, are removed from each graph in $D$; (2) The edges, which are not used as an embedding of a single-edge FAS, are removed from each graph in $D$.

Theorem 3. Let $L_{U}^{V} \subseteq L_{V}$ be the label set used by the single-vertex FASs in a collection $D$ and $u$ be a vertex with label $l_{u} \in L_{V}$. The following statements are true:

1. The vertex $u$ is not used as an embedding by any single-vertex FAS if and only if $L_{U}^{V} \cap U_{V}^{\tau}\left(l_{u}\right)=\emptyset$.

2. If $L_{U}^{V} \cap U_{V}^{\tau}\left(l_{u}\right)=\emptyset$ then a non-frequent subgraph is obtained when $e=\{v, u\}$ extends an existing FAS $G$ in $D$, where $u$ is the new vertex of this forward extension.

Proof. First, we will prove statement 1. Suppose that the vertex $u$ is used as an embedding by a single-vertex FAS $Y=\left(\{w\}, \emptyset, I_{Y}, J_{Y}\right)$. If this occurs, then the label $l_{w} \in L_{U}^{V}$ of the vertex $w$ replaces the label $l_{u}$ with a value greater than or equal to $\tau$, i.e. $l_{w} \in U_{V}^{\tau}\left(l_{u}\right)$, and $L_{U}^{V} \cap U_{V}^{\tau}\left(l_{u}\right) \neq \emptyset$. Therefore, if $L_{U}^{V} \cap U_{V}^{\tau}\left(l_{u}\right)=\emptyset$, then the vertex $u$ is not used as an embedding by any single-vertex FAS. On the other hand, assuming that $L_{U}^{V} \cap U_{V}^{\tau}\left(l_{u}\right) \neq \emptyset$, then $u$ is not used as an embedding by any single-vertex FAS because their labels replace $l_{u}$ with values less than $\tau$. Therefore, if $u$ is used as an embedding by a single-vertex FAS, then $L_{U}^{V} \cap U_{V}^{\tau}\left(l_{u}\right)=\emptyset$.

Next, we will prove the second statement. Suppose that we have a forward extension $e=\{v, u\}$, with $u$ as the new vertex, of an existing FAS $G$ in $D$. 
If $L_{U}^{V} \cap U_{V}^{\tau}\left(l_{u}\right)=\emptyset$ then the vertex $u$ is not used as an embedding by any single-vertex FAS, according to the first part of the Theorem and, as a directly consequence, the single-vertex subgraph $T=\left(\{u\}, \emptyset, I_{T}, J_{T}\right)$ is non-frequent. As $T \subseteq{ }_{A} G \diamond e$, applying the downward closure, we have $\operatorname{supp}(G \diamond e, D) \leq \operatorname{supp}(T, D)$, then $G \diamond e$ is a non-frequent pattern because $T$ is a non-frequent. Therefore, to prune the vertices with label $l_{u}$ of $D$ does not change the frequent pattern output.

Theorem 4. Let $L_{U}^{E} \subseteq L_{E}$ be the label set used by the single-edge FASs in a collection $D$ and an edge $e$ with label $l_{e} \in L_{E}$, the following statements are true:

1. The edge $e$ is not used as an embedding by any single-edge FAS if and only if $L_{U}^{E} \cap U_{E}^{\tau}\left(l_{e}\right)=\emptyset$.

2. If $L_{U}^{E} \cap U_{E}^{\tau}\left(l_{e}\right)=\emptyset$ then a non-frequent subgraph is obtained if e extends a $F A S G$ in $D$.

Proof. First, we will prove statement 1. Suppose that the edge $e$ is used as an embedding by a single-edge FAS $Y=\left(V_{Y},\left\{e^{\prime}\right\}, I_{Y}, J_{Y}\right)$. If this occurs, then the label $l_{e^{\prime}} \in L_{U}^{E}$ of the edge $e^{\prime}$ replaces the label $l_{e}$ with a value greater than or equal to $\tau$, i.e. $l_{e^{\prime}} \in U_{V}^{\tau}\left(l_{e}\right)$, and $L_{U}^{E} \cap U_{E}^{\tau}\left(l_{e}\right) \neq \emptyset$. Therefore, if $L_{U}^{E} \cap U_{E}^{\tau}\left(l_{e}\right)=\emptyset$, then the edge $e$ is not used as an embedding by any single-edge FAS. On the other hand, assuming that $L_{U}^{E} \cap U_{E}^{\tau}\left(l_{e}\right) \neq \emptyset$, then $e$ is not used as an embedding by any single-edge FAS because their labels replace $l_{e}$ with values less than $\tau$. Therefore, if $e$ is used as an embedding by a single-edge FAS, then $L_{U}^{E} \cap U_{E}^{\tau}\left(l_{e}\right)=\emptyset$.

Next, we will prove the second statement. Suppose that we have an extension $e$ of an existing FAS $G$ in $D$. If $L_{U}^{E} \cap U_{E}^{\tau}\left(l_{e}\right)=\emptyset$ then the edge $e$ is not used as an embedding by any single-edge FAS, according to the first part of the Theorem and, as a directly consequence, the single-edge subgraph $T$ with $l_{e}$ as edge label is non-frequent. As $T \subseteq_{A} G \diamond e$, applying the downward closure, we have $\operatorname{supp}(G \diamond e, D) \leq \operatorname{supp}(T, D)$, then $G \diamond e$ is a non-frequent pattern because $T$ is a non-frequent. Therefore, to prune the edges with label $l_{e}$ of $D$ does not change the frequent pattern output.

VEAM and APGM are shown through three pseudo-codes where the common main algorithm consists in finding the single-vertex FAS and single-edge FAS sets, then the first set is stored in set $F$ and only the second one is stored in set $C$ (see Algorithm 1 in Figure 1). A prune using Theorem 3 as basis is performed in line 2. After obtaining the single-edge FAS set, a prune using Theorem 4 as basis is performed in line 5. As we can see, in order to apply the last prune, a breadth-first search (BFS) is required to obtain the approximate frequent edges in the initial process. Later, for each pattern in $C$ the algorithm Search is invoked. When all single-edge FASs in $C$ have been extended, then the set $F$ of all FASs in $D$ is returned.

The other common pseudo-code of VEAM and APGM performs the extension of subgraph patterns on one edge using DFS (see Algorithm 2 in Figre 1). Thus, all candidate subgraphs are created using the label set obtained through the "appLSetVEAM" algorithm, in the VEAM case, or "appLSetAPGM" algorithm, in the APGM case, in line 2. In Algorithm 3 (see lines 1 and 4) and 
in Algorithm 4 (see line 3), a prune using theorem 2 as basis is included, where only the labels in $U_{E}^{\tau}(J(e))$ and $U_{V}^{\tau}(I(v))$ are used.

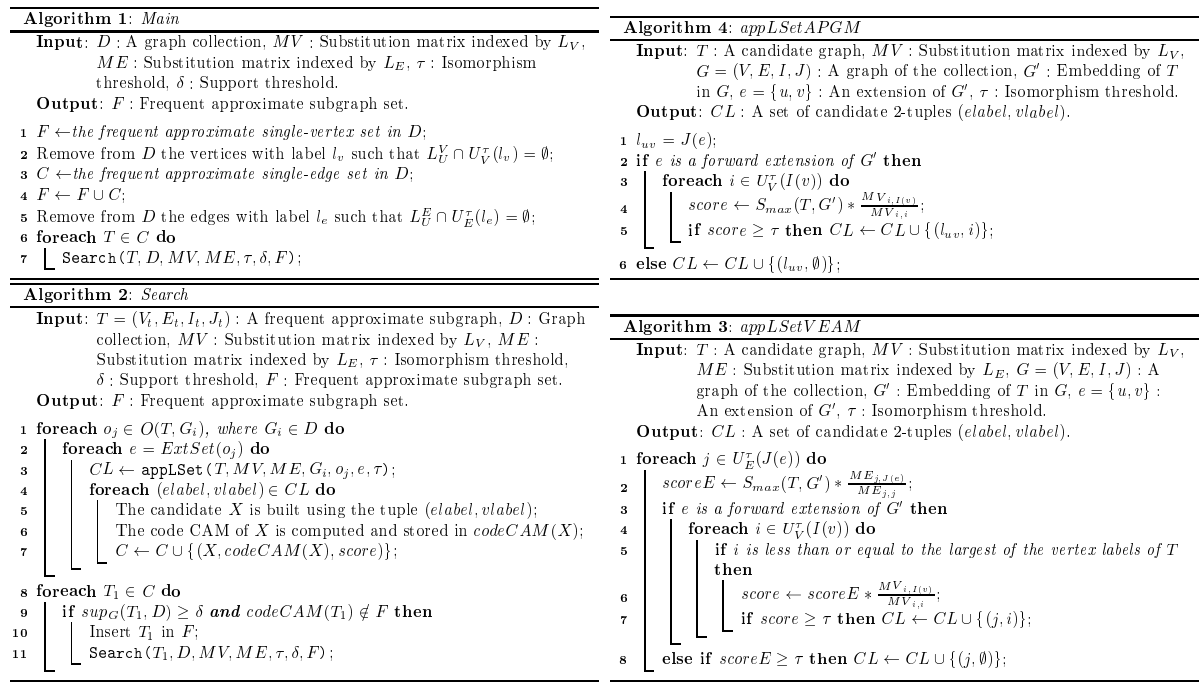

Fig. 1. Pseudo-code of VEAM and APGM using the proposed prunes

\section{Experimental Results}

All our experiments were carried out using a personal computer ( $x 64$ platform) Intel (R) Core (TM) 2 Quad CPU Q9450 @ 2.66 GHz with 4 Gb main memory. We used ANSI $\mathrm{C}$ language and we compiled the algorithms using gcc compiler of GNU/Linux.

In this paper, the mining process is performed in the several image collections used by Acosta-Mendoza et al. [1]. The impact of our prunes in FAS mining on image collections is evaluated with the aim of showing the usefulness of these prunes. The performance of VEAM and APGM with and without our prunes in several collections are compared as follows. Note that in Table 1 are shown only the results of the algorithms in two collections (D700 and D600) due to space restrictions.

First, VEAM and APGM with and without the prunes proposed are compared regarding the number of exhaustive CF-tests that they perform in their mining strategies (see subtable a) in Table 1). Note that the original algorithms are denoted by VEAM and APGM, and the algorithms with our prunes are denoted by $V E A M w P$ and $A P G M w P$. These CF-tests become much more complex to great extent with the increase of the candidate size. In this comparison, the number

${ }^{4}$ The APGM version used in our experiments was implemented by us to detect all frequent vertex approximate subgraphs and not just the clique subgraphs as proposed [6]. 
of such expensive CF-tests, in most of the cases, were reduced in $30 \%$ when our prunes are used. The fact that these prunes reduce the number of candidates to be processed impacts in a positive manner in the algorithm's performance.

Second, the performance, in terms of runtime, of APGM and VEAM with and without the prunes proposed are compared. In this comparison the runtimes were reduced, in most of the cases, in $15 \%$, when our prunes are used. Notice that we use the same support threshold and isomorphism threshold values presented by Acosta-Mendoza et al. [1] with the purpose of showing the improvement achieving the same accuracies obtained by them.

Table 1. Comparison between VEAM and VEAMwP, and between APGM and APGMwP in image collections using $\tau=0.4$

a) Number of CF-tests computed

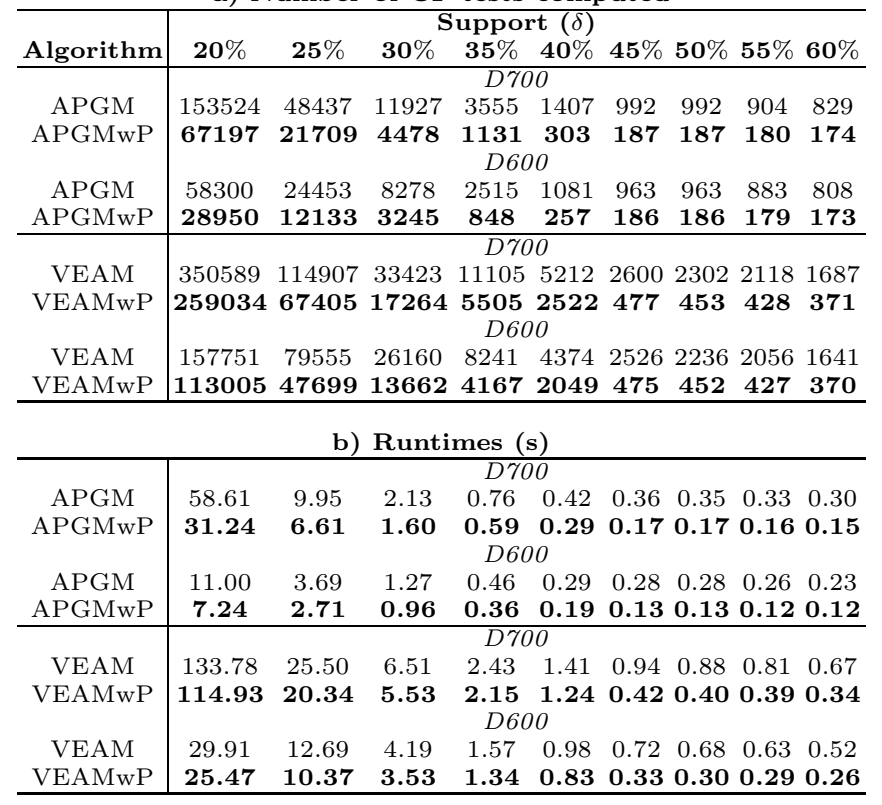

In summary, we can conclude that our prunes positively impact the performance of APGM and VEAM. The prune based on Theorem 2 is very effective when the number of dissimilarities between labels or the number of candidates are increased. The prune using Theorems 3 and 4 is considered effective when the collection has many non-frequent vertices and edges, and its graphs are dense. In general, the new prunes help us to reduce runtimes of VEAM and APGM. These results ensure the usefulness of the prunes proposed in this paper.

\section{Conclusions and Future Work}

In this paper, we introduced a prune which is useful to reduce the number of CF-tests during the FAS mining. This prune allows decreasing the search space 
of collection's graphs. Another prune, which uses only the label set that comply with the isomorphism threshold, was proposed. These prunes are implemented and tested in already reported FAS miners, APGM and VEAM. As can be seen in the experimental results, the number of CF-tests is notably reduced, and the improvement in time is appreciable.

As future work, we are going to develop new ways for taking advantage of the candidate reduction in order to achieve better performance in FAS mining. This allows us to accelerate the support calculation by the reduction of the number of test sub-isomorphism, and in turn, the number of embedded to keep.

\section{References}

1. Acosta-Mendoza, N., Gago-Alonso, A., Medina-Pagola, J.E.: Frequent Approximate Subgraphs as Features for Graph-Based Image Classification. KnowledgeBased Systems 27, 381-392 (2012)

2. Agrawal, R., Srikant, R.: Fast Algorithms for Mining Association Rules. In: In Proceedings of the 1994 International Conference on Very Large Data Bases (VLDB 1994), Santiago, Chile, pp. 487-499 (1994)

3. Borgelt, C.: Canonical Forms for Frequent Graph Mining. In: Proceedings of the 30th Annual Conference of the Gesellschaft für Klassifikation e.V., pp. 8-10. Universitat Berlin (2006)

4. Chen, C., Yan, X., Zhu, F., Han, J.: gApprox: Mining Frequent Approximate Patterns from a Massive Network. In: IEEE International Conference on Data Mining, ICDM 2007, pp. 445-450 (2007)

5. Holder, L.B., Cook, D.J., Bunke, H.: Fuzzy substructure discovery. In: Proceedings of the 9th International Workshop on Machine Learning, San Francisco, CA, USA, pp. 218-223 (1992)

6. Jia, Y., Zhang, J., Huan, J.: An Efficient Graph-Mining Method for Complicated and Noisy Data with Real-World Applications. Knowledge Information Systems 28(2), 423-447 (2011)

7. Jiang, C., Coenen, F., Zito, M.: A Survey of Frequent Subgraph Mining Algorithms. To appear: Knowledge Engineering Review (2012)

8. Song, Y., Chen, S.: Item Sets Based Graph Mining Algorithm and Application in Genetic Regulatory Networks. In: Proceedings of the IEEE International Conference on Granular Computing, Atlanta, GA, USA, pp. 337-340 (2006)

9. Xiao, Y., Wu, W., Wang, W., He, Z.: Efficient Algorithms for Node Disjoint Subgraph Homeomorphism Determination. In: Haritsa, J.R., Kotagiri, R., Pudi, V. (eds.) DASFAA 2008. LNCS, vol. 4947, pp. 452-460. Springer, Heidelberg (2008)

10. Zhang, S., Yang, J.: RAM: Randomized Approximate Graph Mining. In: Ludäscher, B., Mamoulis, N. (eds.) SSDBM 2008. LNCS, vol. 5069, pp. 187-203. Springer, Heidelberg (2008)

11. Zou, Z., Li, J., Gao, H., Zhang, S.: Mining Frequent Subgraph Patterns from Uncertain Graph Data. IEEE Transactions on Knowledge and Data Engineering 22(9), 1203-1218 (2010) 